CREAT. MATH. INFORM.

Volume 25 (2016), No. 2,

Pages 215 - 222
Online version at https : //creative-mathematics. cunbm. utcluj.ro/

Print Edition: ISSN 1584 - 286X; Online Edition: ISSN 1843 - 441X

DOI: https://doi.org/10.37193/CMI.2016.02.13

\title{
Multiplicity of positive solutions for second order Sturm-Liouville boundary value problems
}

\author{
K. R. PRASAD ${ }^{1}$, N. SREEDHAR ${ }^{2}$ and L. T. WESEN ${ }^{3}$
}

ABSTRACT. In this paper, we develop criteria for the existence of multiple positive solutions for second order Sturm-Liouville boundary value problem,

$$
\begin{gathered}
u^{\prime \prime}+k^{2} u+f(t, u)=0,0 \leq t \leq 1, \\
a u(0)-b u^{\prime}(0)=0 \text { and } c u(1)+d u^{\prime}(1)=0,
\end{gathered}
$$

where $k \in\left(0, \frac{\pi}{2}\right)$ is a constant, by an application of Avery-Henderson fixed point theorem.

\section{INTRODUCTION}

The existence of positive solutions of the boundary value problems have been studied by many researchers due to the importance in both theory and applications. In recent years, there is an increasing interest shown in establishing the existence of positive solutions for second order Neumann and Sturm-Liouville boundary value problems associated with ordinary differential equations by applying various techniques. These type of problems arise in modeling of different areas of applied mathematics, physics and biological sciences.

By using the fixed point theorems in cones, Jiang and Liu [7], Sun and Li [13], Sun, $\mathrm{Li}$ and Cheng [14], Li and Jiang [9], Yao [23], Chu, Sun and Chen [3], Wang, Cui and Zhang [21], Sun, Cho and O'Regan [12], Wang and Zhang [22], Li, Cong, Li and Lv [8] and Henderson and Kosmatov [4] have studied the existence of positive solutions for second order Neumann boundary value problems.

Tian and Ge $[16,17]$ considered the general Sturm-Liouville problems and established the existence of positive solutions for second order Sturm-Liouville boundary value problem with a $p$-Laplacian by using critical point theory. For the back ground, results and some recent contributions, we refer $[1,5,6,10,11,15,18,19,20]$.

Motivated by the papers mentioned above, in this paper, we establish the existence of even number of positive solutions for second order differential equations of the form,

$$
u^{\prime \prime}+k^{2} u+f(t, u)=0,0 \leq t \leq 1,
$$

satisfying the Sturm-Liouville boundary conditions,

$$
a u(0)-b u^{\prime}(0)=0 \text { and } c u(1)+d u^{\prime}(1)=0,
$$

where $k \in\left(0, \frac{\pi}{2}\right)$ is a constant, $a, b, c$ and $d$ are positive constants such that $k^{2} \leq \frac{a c}{b d}$ and $f:[0,1] \times \mathbb{R}^{+} \rightarrow \mathbb{R}^{+}$is a continuous function, by an application of Avery-Henderson fixed point theorem.

Received: 28.02.2016. In revised form: 06.05.2016. Accepted: 13.05.2016

2010 Mathematics Subject Classification. 34B15.

Key words and phrases. Green's function, boundary value problem, positive solution, cone.

Corresponding author: N. Sreedhar; sreedharnamburi@rediffmail.com 
The rest of the paper is organized as follows. In Section 2, we construct the Green's function for the homogeneous problem corresponding to (1.1)-(1.2) and estimate bounds for the Green's function. In Section 3, we establish a criteria for the existence of at least two positive solutions for the boundary value problem (1.1)-(1.2) by using an AveryHenderson fixed point theorem [2]. And then, we establish the existence of at least $2 n$ positive solutions to the boundary value problem (1.1)-(1.2) for an arbitrary positive integer $n$. Finally as an application, we give an example to illustrate our result.

\section{GREEN'S FUNCTION AND BOUNDS}

In this section, we construct the Green's function for the homogeneous problem corresponding to (1.1)-(1.2) and estimate bounds for the Green's function.

Let $G(t, s)$ be the Green's function for the homogeneous problem,

$$
-\left(u^{\prime \prime}+k^{2} u\right)=0,0 \leq t \leq 1,
$$

satisfying the boundary conditions (1.2).

Lemma 2.1. Let $d=k\left(a c-b d k^{2}\right) \sin k+k^{2}(a d+b c) \cos k \neq 0$. Then the Green's function $G(t, s)$ for the boundary value problem (2.3), (1.2) is given by

$$
G(t, s)=\left\{\begin{array}{l}
\frac{1}{d}(a \sin k t+b k \cos k t)(c \sin k(1-s)+d k \cos k(1-s)), t \leq s \\
\frac{1}{d}(a \sin k s+b k \cos k s)(c \sin k(1-t)+d k \cos k(1-t)), s \leq t
\end{array}\right.
$$

Lemma 2.2. The Green's function $G(t, s)$ satisfies the following inequalities:

(i) $G(t, s)>0$, for all $t, s \in(0,1)$,

(ii) $G(t, s) \leq M G(s, s)$, for all $(t, s) \in[0,1] \times[0,1]$,

(iii) $\frac{1}{M} G(s, s) \leq G(t, s)$, for all $(t, s) \in[0,1] \times[0,1]$,

where $M=\max \left\{\frac{a+b k}{b k \cos k}, \frac{c+d k}{d k \cos k}\right\}$.

Proof. (i) Since $k^{2} \leq \frac{a c}{b d}$, the Green's function $G(t, s)$ is positive for all $t, s \in(0,1)$.

(ii) Let $t \leq s$. Then

$$
\frac{G(t, s)}{G(s, s)}=\frac{a \sin k t+b k \cos k t}{a \sin k s+b k \cos k s} \leq \frac{a+b k}{b k \cos k}
$$

Let $s \leq t$. Then

$$
\frac{G(t, s)}{G(s, s)}=\frac{c \sin k(1-t)+d k \cos k(1-t)}{c \sin k(1-s)+d k \cos k(1-s)} \leq \frac{c+d k}{d k \cos k}
$$

Therefore,

$$
G(t, s) \leq M G(s, s), \text { for all }(t, s) \in[0,1] \times[0,1],
$$

where $M=\max \left\{\frac{a+b k}{b k \cos k}, \frac{c+d k}{d k \cos k}\right\}$.

(iii) We can prove the inequality (iii) as in (ii). 


\section{Multiple POSITIVE SOLUTIONS}

In this section, we establish the existence of at least two positive solutions for the boundary value problem (1.1)-(1.2) by using an Avery-Henderson functional fixed point theorem. And then, we establish the existence of at least $2 n$ positive solutions to the boundary value problem (1.1)-(1.2) for an arbitrary positive integer $n$.

Let $B$ be a real Banach space. A nonempty closed convex set $P \subset B$ is called a cone, if it satisfies the following two conditions:

(i) $y \in P, \lambda \geq 0$ implies $\lambda y \in P$, and

(ii) $y \in P$ and $-y \in P$ implies $y=0$.

Let $\psi$ be a nonnegative continuous functional on a cone $P$ of the real Banach space $B$. Then for a positive real number $c^{\prime}$, we define the sets

$$
P\left(\psi, c^{\prime}\right)=\left\{y \in P: \psi(y)<c^{\prime}\right\}
$$

and

$$
P_{a^{\prime}}=\left\{y \in P:\|y\|<a^{\prime}\right\} .
$$

In obtaining multiple positive solutions of the boundary value problem (1.1)-(1.2), the following Avery-Henderson functional fixed point theorem will be the fundamental tool.

Theorem 3.1. [2] Let $P$ be a cone in a real Banach space B. Suppose $\alpha$ and $\gamma$ are increasing, nonnegative continuous functionals on $P$ and $\theta$ is nonnegative continuous functional on $P$ with $\theta(0)=0$ such that, for some positive numbers $c^{\prime}$ and $k, \gamma(y) \leq \theta(y) \leq \alpha(y)$ and $\|y\| \leq k \gamma(y)$, for all $y \in \overline{P\left(\gamma, c^{\prime}\right)}$. Suppose that there exist positive numbers $a^{\prime}$ and $b^{\prime}$ with $a^{\prime}<b^{\prime}<c^{\prime}$ such that $\theta(\lambda y) \leq \lambda \theta(y)$, for all $0 \leq \lambda \leq 1$ and $y \in \partial P\left(\theta, b^{\prime}\right)$. Further, let $T: \overline{P\left(\gamma, c^{\prime}\right)} \rightarrow P$ be $a$ completely continuous operator such that

(B1) $\gamma(T y)>c^{\prime}$, for all $y \in \partial P\left(\gamma, c^{\prime}\right)$,

(B2) $\theta(T y)<b^{\prime}$, for all $y \in \partial P\left(\theta, b^{\prime}\right)$,

(B3) $P\left(\alpha, a^{\prime}\right) \neq \emptyset$ and $\alpha(T y)>a^{\prime}$, for all $y \in \partial P\left(\alpha, a^{\prime}\right)$.

Then, $T$ has at least two fixed points $y_{1}, y_{2} \in \overline{P\left(\gamma, c^{\prime}\right)}$ such that $a^{\prime}<\alpha\left(y_{1}\right)$ with $\theta\left(y_{1}\right)<b^{\prime}$ and $b^{\prime}<\theta\left(y_{2}\right)$ with $\gamma\left(y_{2}\right)<c^{\prime}$.

Let $B=\{u: u \in C[0,1]\}$ be the Banach space equipped with the norm

$$
\|u\|=\max _{t \in[0,1]}|u(t)| \text {. }
$$

Define the cone $P \subset B$ by

$$
P=\left\{u \in B: u(t) \geq 0 \text { on }[0,1] \text { and } \min _{t \in[0,1]} u(t) \geq m\|u\|\right\}
$$

where $m=\frac{1}{M^{2}}$.

Define the nonnegative, increasing, continuous functionals $\gamma, \theta$ and $\alpha$ on the cone $P$ by

$$
\gamma(u)=\min _{t \in[0,1]} u(t), \theta(u)=\max _{t \in[0,1]} u(t) \text { and } \alpha(u)=\max _{t \in[0,1]} u(t) .
$$

We observe that for any $u \in P$,

$$
\gamma(u) \leq \theta(u)=\alpha(u)
$$

and

$$
\|u\| \leq \frac{1}{m} \min _{t \in[0,1]} u(t)=\frac{1}{m} \gamma(u) \leq \frac{1}{m} \theta(u)=\frac{1}{m} \alpha(u) .
$$

Define

$$
L=\int_{0}^{1} G(s, s) d s .
$$


Theorem 3.2. Suppose there exist real numbers $a^{\prime}, b^{\prime}$ and $c^{\prime}$ with $0<a^{\prime}<b^{\prime}<c^{\prime}$ such that $f$ satisfies the following conditions:

(D1) $f(t, u)>\frac{M c^{\prime}}{b_{b^{\prime}}}$, for $t \in[0,1]$ and $u \in\left[c^{\prime}, \frac{c^{\prime}}{b^{\prime}}\right]$,

(D2) $f(t, u)<\frac{b^{\prime}}{M L}$, for $t \in[0,1]$ and $u \in\left[0, \frac{b^{\prime}}{m}\right]$,

(D3) $f(t, u)>\frac{M a^{\prime}}{L}$, for $t \in[0,1]$ and $u \in\left[a^{\prime}, \frac{a^{\prime}}{m}\right]$.

Then the boundary value problem (1.1)-(1.2) has at least two positive solutions $u_{1}$ and $u_{2}$ such that

$$
\begin{gathered}
a^{\prime}<\max _{t \in[0,1]} u_{1}(t) \text { with } \max _{t \in[0,1]} u_{1}(t)<b^{\prime}, \\
b^{\prime}<\max _{t \in[0,1]} u_{2}(t) \text { with } \min _{t \in[0,1]} u_{2}(t)<c^{\prime} .
\end{gathered}
$$

Proof. Define the operator $T: P \rightarrow B$ by

$$
T u(t)=\int_{0}^{1} G(t, s) f(s, u(s)) d s .
$$

It is obvious that a fixed point of $T$ is the solution of the boundary value problem (1.1)(1.2). We seek two fixed points $u_{1}, u_{2} \in P$ of $T$. First, we show that $T: P \rightarrow P$. Let $u \in P$. From Lemma 2.2, we have $T u(t) \geq 0$ on $[0,1]$ and also,

$$
\begin{aligned}
T u(t) & =\int_{0}^{1} G(t, s) f(s, u(s)) d s \\
& \leq M \int_{0}^{1} G(s, s) f(s, u(s)) d s
\end{aligned}
$$

so that

$$
\|T u\| \leq M \int_{0}^{1} G(s, s) f(s, u(s)) d s .
$$

Next, if $u \in P$, then we have

$$
\begin{aligned}
T u(t) & =\int_{0}^{1} G(t, s) f(s, u(s)) d s \\
& \geq \frac{1}{M} \int_{0}^{1} G(s, s) f(s, u(s)) d s \\
& \geq \frac{1}{M^{2}}\|T u\|=m\|T u\| .
\end{aligned}
$$

Hence $T u \in P$ and so $T: P \rightarrow P$. Moreover, $T$ is completely continuous. From (3.5) and (3.6), for each $u \in P$, we have $\gamma(u) \leq \theta(u)=\alpha(u)$ and $\|u\| \leq \frac{1}{m} \gamma(u)$. Also, for any $0 \leq \lambda \leq 1$ and $u \in P$, we have $\theta(\lambda u)=\max _{t \in[0,1]}(\lambda u)(t)=\lambda \max _{t \in[0,1]} u(t)=\lambda \theta(u)$. It is clear that $\theta(0)=0$. We now show that the remaining conditions of Theorem 3.1 are satisfied.

Firstly, we shall verify that condition $(B 1)$ of Theorem 3.1 is satisfied. Since $u \in \partial P\left(\gamma, c^{\prime}\right)$, 
from (3.6) we have that $c^{\prime}=\min _{t \in[0,1]} u(t) \leq\|u\| \leq \frac{c^{\prime}}{m}$. Then

$$
\begin{aligned}
\gamma(T u) & =\min _{t \in[0,1]} \int_{0}^{1} G(t, s) f(s, u(s)) d s \\
& \geq \frac{1}{M} \int_{0}^{1} G(s, s) f(s, u(s)) d s \\
& >\frac{c^{\prime}}{L} \int_{0}^{1} G(s, s) d s=c^{\prime},
\end{aligned}
$$

using hypothesis $(D 1)$.

Now we shall show that condition $(B 2)$ of Theorem 3.1 is satisfied. Since $u \in \partial P\left(\theta, b^{\prime}\right)$, from (3.6) we have that $0 \leq u(t) \leq\|u\| \leq \frac{b^{\prime}}{m}$, for $[0,1]$. Thus

$$
\begin{aligned}
\theta(T u) & =\max _{t \in[0,1)]} \int_{0}^{1} G(t, s) f(s, u(s)) d s \\
& \leq M \int_{0}^{1} G(s, s) f(s, u(s)) d s \\
& <\frac{b^{\prime}}{L} \int_{0}^{1} G(s, s) d s=b^{\prime}
\end{aligned}
$$

by hypothesis $(D 2)$.

Finally, using hypothesis $(D 3)$, we shall show that condition $(B 3)$ of Theorem 3.1 is satisfied. Since $0 \in P$ and $a^{\prime}>0, P\left(\alpha, a^{\prime}\right) \neq \emptyset$. Since $u \in \partial P\left(\alpha, a^{\prime}\right), a^{\prime}=\max _{t \in[0,1]} u(t) \leq$ $\|u\| \leq \frac{a^{\prime}}{m}$, for $t \in[0,1]$. Therefore,

$$
\begin{aligned}
\alpha(T u) & =\max _{t \in[0,1]} \int_{0}^{1} G(t, s) f(s, u(s)) d s \\
& \geq \frac{1}{M} \int_{0}^{1} G(s, s) f(s, u(s)) d s \\
& >\frac{a^{\prime}}{L} \int_{0}^{1} G(s, s) d s=a^{\prime} .
\end{aligned}
$$

Thus, all the conditions of Theorem 3.1 are satisfied and so there exist at least two positive solutions $u_{1}, u_{2} \in \overline{P\left(\gamma, c^{\prime}\right)}$ for the boundary value problem (1.1)-(1.2). This completes the proof of the theorem.

Theorem 3.3. Let $n$ be an arbitrary positive integer. Assume that there exist numbers $a_{r}(r=$ $1,2, \cdots, n+1)$ and $b_{s}(s=1,2, \cdots, n)$ with $0<a_{1}<b_{1}<a_{2}<b_{2}<\cdots<a_{n}<b_{n}<a_{n+1}$ such that

$$
\begin{gathered}
f(t, u)>\frac{M a_{r}}{L}, \text { for } t \in[0,1] \text { and } u \in\left[a_{r}, \frac{a_{r}}{m}\right], r=1,2, \cdots, n+1, \\
f(t, u)<\frac{b_{s}}{M L}, \text { for } t \in[0,1] \text { and } u \in\left[0, \frac{b_{s}}{m}\right], s=1,2, \cdots, n .
\end{gathered}
$$

Then the boundary value problem (1.1)-(1.2) has at least $2 n$ positive solutions in $\bar{P}_{a_{n+1}}$.

Proof. We use induction on $n$. For $n=1$, we know from (3.8) and (3.9) that $T: \bar{P}_{a_{2}} \rightarrow$ $P_{a_{2}}$, then, it follows from Avery-Henderson fixed point theorem that the boundary value problem (1.1)-(1.2) has at least two positive solutions in $\bar{P}_{a_{2}}$. Next, we assume that this conclusion holds for $n=l$. In order to prove this conclusion holds for $n=l+1$. We 
suppose that there exist numbers $a_{r}(r=1,2, \cdots, l+2)$ and $b_{s}(s=1,2, \cdots, l+1)$ with $0<a_{1}<b_{1}<a_{2}<b_{2}<\cdots<a_{l+1}<b_{l+1}<a_{l+2}$ such that

$$
\begin{gathered}
f(t, u)>\frac{M a_{r}}{L}, \text { for } t \in[0,1] \text { and } u \in\left[a_{r}, \frac{a_{r}}{m}\right], r=1,2, \cdots, l+2, \\
f(t, u)<\frac{b_{s}}{M L}, \text { for } t \in[0,1] \text { and } u \in\left[0, \frac{b_{s}}{m}\right], s=1,2, \cdots, l+1 .
\end{gathered}
$$

By assumption, the BVP (1.1)-(1.2) has at least $2 l$ positive solutions $u_{i}(i=1,2, \cdots, 2 l)$ in $\bar{P}_{a_{l+1}}$. At the same time, it follows from Theorem 3.2, (3.10) and (3.11) that the boundary value problem (1.1)-(1.2) has at least two positive solutions $u_{1}, u_{2}$ in $\bar{P}_{a_{l+2}}$ such that $a_{l+1}<$ $\alpha\left(u_{1}\right)$ with $\theta\left(u_{1}\right)<b_{l+1}$ and $b_{l+1}<\theta\left(u_{2}\right)$ with $\gamma\left(u_{2}\right)<a_{l+2}$. Obviously $u_{1}$ and $u_{2}$ are different from $u_{i}(i=1,2, \cdots, 2 l)$. Therefore, the boundary value problem (1.1)-(1.2) has at least $2 l+2$ positive solutions in $\bar{P}_{a_{l+2}}$, which shows that the conclusion holds for $n=$ $l+1$.

Example 3.1. Let us consider an example to illustrate the usage of the Theorem 3.2. Now, consider the following problem,

$$
u^{\prime \prime}+k^{2} u+f(t, u)=0,0 \leq t \leq 1,
$$

subject to the boundary conditions,

$$
2 u(0)-u^{\prime}(0)=0 \text { and } 3 u(1)+2 u^{\prime}(1)=0,
$$

where

$$
f(t, u)=\frac{10(u+1)^{5}}{73\left(u^{2}+99\right)} .
$$

The Green's function $G(t, s)$ for the homogeneous problem,

$$
-\left(u^{\prime \prime}+k^{2} u\right)=0,0 \leq t \leq 1,
$$

satisfying the boundary conditions (3.13) is given by

$$
G(t, s)=\left\{\begin{array}{l}
\frac{(2 \sin k t+k \cos k t)(3 \sin k(1-s)+2 k \cos k(1-s))}{5.7002669}, t \leq s, \\
\frac{(2 \sin k s+k \cos k s)(3 \sin k(1-t)+2 k \cos k(1-t))}{5.7002669}, s \leq t .
\end{array}\right.
$$

By algebraic computations, we get

$$
M=2.015478827, m=0.03975348409, L=0.3977489636 .
$$

Clearly $f$ is continuous and increasing on $[0, \infty)$. If we choose $a^{\prime}=0.0001, b^{\prime}=0.01$ and $c^{\prime}=150$ then $0<a^{\prime}<b^{\prime}<c^{\prime}$ and $f$ satisfies

(i) $f(t, u)>1891.448861=\frac{M c^{\prime}}{L}$, for $t \in[0,1]$ and $u \in[150,3773.25418]$,

(ii) $f(t, u)<0.0042462538=\frac{b^{\prime}}{M L}$, for $t \in[0,1]$ and $u \in[0,0.2515502786]$,

(iii) $f(t, u)>0.0013843920=\frac{M a^{\prime}}{L}$, for $t \in[0,1]$ and $u \in[0.0001,0.002515502]$.

Then all the conditions of Theorem 3.2 are satisfied. Thus by Theorem 3.2, the boundary value problem (3.12)-(3.13) has at least two positive solutions $u_{1}$ and $u_{2}$ satisfying

$$
0.0001<\max _{t \in[0,1]} u_{1}(t) \text { with } \max _{t \in[0,1]} u_{1}(t)<0.01,
$$




$$
0.01<\max _{t \in[0,1]} u_{2}(t) \text { with } \min _{t \in[0,1]} u_{2}(t)<150 .
$$

Acknowledgements. The authors thank the referees for their valuable suggestions and comments.

\section{REFERENCES}

[1] Agarwal, R. P., Hong, H. L. and Yeh, C. C., The existence of positive solutions for the Strum-Liouville boundary value problems, Comp. Math. Appl., 35 (1998), No. 9, 89-96

[2] Avery, R. I. and Henderson, J., Two positive fixed points of nonlinear operators on ordered Banach spaces, Comm. Appl. Nonlinear Anal., 8 (2001), 27-36

[3] Chu, J., Sun Y. and Chen, H., Positive solutions of Neumann problems with singularities, J. Math. Anal. Appl., 337 (2008), 1267-1272

[4] Henderson, J. and Kosmatov, N., Positive solutions of the semipositone Neumann boundary value problem, Math. Model. Anal., 20 (2015), No. 5, 578-584

[5] Il'in, V. A. and Moiseev, E. I., Nonlocal boundary value problem of the first kind for a Sturm-Liouville operator in its differential and finite difference aspects, Diff. Eqns., 23 (1987), No. 7, 803-810

[6] Il'in, V. A. and Moiseev, E. I., Nonlocal boundary value problem of the second kind for a Sturm-Liouville operator, Diff. Eqns., 23 (1987), No. 8, 979-987

[7] Jiang, D. and Liu, H., Existence of positive solutions to second order Neumann boundary value problems, J. Math. Res. Exposition, 20 (2000), No. 3, 360-364

[8] Li, Q., Cong, F., Li, Z. and Lv, J., Multiplicity of positive solutions of superlinear semi-positone singular Neumann problems, Bound. Value Probl., 2014 (2014), No. 217, 1-11

[9] Li, X. and Jiang, D., Optimal existence theory for single and multiple positive solutions to second order Neumann boundary value problems, Indian J. Pure Appl. Math., 35 (2004), No. 5, 573-586

[10] Ma, R. and Thompson, B., Positive solutions for nonlinear m-point eigenvalue problems, J. Math. Anal. Appl., 297 (2004), No. 1, 24-37

[11] Su, H., Liu, L. and Wu, Y., Positive solutions for Sturm-Liouville boundary value problems in a Banach space, Abstr. Appl. Anal., 2012 (2012), Art. ID 572172, 1-11

[12] Sun, Y., Cho, Y. J. and O'Regan, D., Positive solutions for singular second order Neumann boundary value problems via a cone fixed point theorem, Appl. Math. Comput., 210 (2009), 80-86

[13] Sun, J. P. and Li, W. T., Multiple positive solutions to second order Neumann boundary value problems, Appl. Math. Comput., 146 (2003), No. 1, 187-194

[14] Sun, J. P., Li, W. T. and Cheng, S. S., Three positive solutions for second order Neumann boundary value problems, Appl. Math. Lett., 17 (2004), 1079-1084

[15] Tian, Y. and Ge, W., Multiple solutions for a second-order Sturm-Liouville boundary value problem, Taiwanese J. Math., 11 (2007), No. 4, 975-988

[16] Tian, Y. and Ge, W., Second-order Sturm-Liouville boundary value problem involving the one-dimensional pLaplacian, Rocky Mountain J. Math., 38 (2008), No. 1, 309-327

[17] Tian, Y. and Ge, W., Multiple positive solutions for a second order Sturm- Liouville boundary value problem with a p-Laplacian via variational methods, Rocky Mountain J. Math., 39 (2009), No. 1, 325-342

[18] Tian, Y. and Ge, W., Sign-changing and multiple solutions of the Sturm-Liouville boundary value problem via invariant sets of descending flow, Nonlinear Anal., 74 (2011), No. 16, 5480-5494

[19] Tian, Y. and Ge, W., Multiple solutions of Sturm-Liouville boundary value problem via lower and upper solutions and variational methods, Nonlinear Anal., 74 (2011), No. 17, 6733-6746

[20] Tian, Y. and Ge, W., Multiple solutions of impulsive Sturm-Liouville boundary value problem via lower and upper solutions and variational methods, J. Math. Anal. Appl., 387 (2012), No. 2, 475-489

[21] Wang, F., Cui, Y. and Zhang, F., A singular second order Neumann boundary value problem with positive solutions, Thai J. Math., 7 (2009), No. 2, 243-257

[22] Wang, F. and Zhang, F., Existence of positive solutions of Neumann boundary value problem via a cone compressionexpansion fixed point theorem of functional type, J. Appl. Math. Comput., 35 (2011), 341-349

[23] Yao, Q. L., Positive solutions of nonlinear second order Neumann boundary value problems, Chinese J. Eng. Math., 23 (2006), No. 5, 939-942

\footnotetext{
${ }^{1,3}$ Department of Applied Mathematics

ANDHRA UNIVERSITY

VISAKHAPATNAM, 530 003, INDIA

Email address: rajendra92@rediffmail.com

Email address: wesen 08 @gmail.com
} 
2 DEPARTMENT OF MATHEMATICS

GITAM UNIVERSITY

VISAKHAPATNAM, 530 045, INDIA

Email address: sreedharnamburierediffmail.com 